\title{
Huntington's Disease Clinical Trials Corner: April 2020
}

\author{
Filipe B. Rodrigues ${ }^{\mathrm{a}, \mathrm{b}, \mathrm{c}}$ and Edward J. Wild ${ }^{\mathrm{a}, *}$ \\ ${ }^{a}$ UCL Huntington's Disease Centre, UCL Queen Square Institute of Neurology, University College London, UK \\ ${ }^{\mathrm{b}}$ Laboratory of Clinical Pharmacology and Therapeutics, Faculdade de Medicina, Universidade de Lisboa, PT, \\ Portugal \\ ${ }^{\mathrm{c}}$ Instituto de Medicina Molecular, Lisbon, PT, Portugal
}

\begin{abstract}
In this edition of the Huntington's Disease Clinical Trials Corner we expand on the UniQure AMT-130 and on the Neurocrine Biosciences KINECT-HD trials, and list all currently registered and ongoing clinical trials in Huntington's disease.
\end{abstract}

Keywords: Huntington disease, clinical trials

\section{INTRODUCTION}

The Huntington's Disease Clinical Trials Corner is a regular section devoted to highlighting ongoing and recently completed clinical trials in Huntington's disease (HD). Clinical trials previously reviewed by the Huntington's Disease Clinical Trials Corner are listed in Table 1.

In this edition, we highlight the UniQure AMT-130 (NCT04120493) [1], and the Neurocrine Biosciences KINECT-HD trial (NCT04102579) [2]. We tabulate all currently registered and ongoing clinical trials in Tables 2 to 4 . For further details on the methodology used, please refer to the first edition of Huntington's Disease Clinical Trials Corner [3].

If you would like to draw attention to specific trials, please feel free to email us at: f.rodrigues@ucl.ac.uk and e.wild@ucl.ac.uk.

\section{ONGOING CLINICAL TRIALS}

A list of all ongoing clinical trials is given in Tables 2, 3 and 4.

\footnotetext{
${ }^{*}$ Correspondence to: Edward J. Wild, UCL Huntington's Disease Centre, Russell Square 10-12, London, WC1B 5EH, UK. E-mail: e.wild@ucl.ac.uk.
}

In addition to the trials covered below, it is worth mentioning that Wave Life Sciences made a preliminary announcement of results from their ongoing PRECISION-HD2 trial (NCT03225846) [4]. This is a phase $1 \mathrm{~b} / 2 \mathrm{a}$ trial investigating WVE-120102, an intrathecal allele-selective antisense oligonucleotide (ASO). When compared with placebo, this drug was shown to reduce CSF mutant huntingtin by $12.4 \%$ (95\% CI 0.40 to 24.58 ), while CSF total huntingtin and neurofilament light (NfL) remained unchanged. Whist statistically significant, this reduction was derived from a comparison of all ASO doses pooled together (mean change from baseline $-6.0 \%$ [95\% CI -9.57 to 4.85$]$ ) against a placebo arm showing a somewhat larger change than might be expected due to disease progression from natural history studies (mean change from baseline $9.5 \%$ [95\% CI 1.77 to 20.38]). The ASO was also considered to be "generally safe and well tolerated among patients receiving doses up to $16 \mathrm{mg}$ ". No results were disclosed about the PRECISION-HD1 trial (NCT03225833) [5], testing WVE-120101, another intrathecal alleleselective ASO targeting a different single-nucleotide polymorphism. As a result, a new $32 \mathrm{mg}$ dosage cohort will added to both trials and further updates are awaited from the broader program [6]. 
Table 1

Clinical trials previously reviewed by the Huntington's Disease Clinical Trials Corner.

IONIS-HTT $_{\mathrm{Rx}}$, RG6042 and tominersen refer to the same molecule

\begin{tabular}{llll}
\hline & Trial name & Intervention & Edition \\
\hline NCT02519036 & IONIS-HTTRx & IONIS-HTT Rx $^{*}$ & September 2017 [3] \\
NCT02215616 & LEGATO-HD & Laquinimod & \\
NCT02197130 & Amaryllis & PF-02545920 & \\
NCT02006472 & PRIDE-HD & Pridopidine & \\
NCT03225833 & PRECISION-HD1 & WVE-120101 & February 2018 [13] \\
NCT03225846 & PRECISION-HD2 & WVE-120102 & \\
NCT01795859 & FIRST-HD & Deutetrabenazine & \\
NCT02481674 & SIGNAL & VX15/2503 & August 2018 [14] \\
NCT00712426 & CREST-E & Creatine & \\
NCT03761849 & GENERATION-HD1 & RG6042* & January 2019 [15] \\
NCT03344601 & PACE-HD & Physical activity & \\
NCT02535884 & HD-DBS & Deep brain stimulation & June 2019 [16] \\
NCT02453061 & TRIHEP3 & Triheptanoin & \\
NCT04120493 & AMT-130 & AAV5-miHTT & April 2020 \\
NCT04102579 & KINECT-HD & Valbenazine & \\
\hline
\end{tabular}

\section{AMT-130 (NCT04120493)}

Study title: A Phase I/II, Randomized, Double-blind, Sham Control Study to Explore Safety, Tolerability, and Efficacy Signals of Multiple Ascending Doses of Striatally-Administered rAAV5-miHTT Total Huntingtin Gene (HTT) Lowering Therapy (AMT-130) in Early Manifest Huntington Disease [1].

Intervention: Single-time intrastriatal injection of AAV5-miHTT [7].

Description: The AMT-130 trial, sponsored by UniQure, aims to evaluate the safety, tolerability and proof-of-concept of a single-time bilateral intrastriatal injection of AAV5-miHTT in adults (25 to 65 years of age) with manifest HD (i.e. clinically symptomatic and genetically confirmed [CAG $\geq 44]$ ) and early disease stage, comparing with sham injection, for disease progression.

Individuals who have received any experimental agent or participation in the following are not eligible for this study: any investigational trial within 60 days or five half-lives prior to screening; with a deep brain stimulator in situ; with history of gene therapy, RNA or DNA targeted HD specific investigational agent, cell transplantation or other experimental cerebral surgery; contraindications for lumbar punctures or 3 Tesla MRI; putaminal and caudate volumes per side inferior to 2.5 and $2.0 \mathrm{~cm}^{3}$, respectively; brain or spinal cord pathology that may interfere with CSF homeostasis and circulation, increased intracranial pressure, malformations or tumours; hospitalization for major medical reason or major surgical proce- dure involving general anaesthesia within 12 weeks of screening; current use of medications to treat or that can aggravate chorea, or unstable concomitant medication within 3 months of screening.

This trial is an US-based, multi-centre, randomized, sham-controlled, double-blind, parallel study. It will have 3 study arms: the low dose group, where participants will receive a single total dose of $6 \times 10^{12}$ genome copies of AAV5-miHTT via a MRI-guided convection-enhanced delivery; the high dose group, where participants will receive a single total dose of $6 \times 10^{13}$ genome copies of AAV5-miHTT via a MRI-guided convection-enhanced delivery; and the imitation surgery arm, where participants will receive bilateral partial thickness burr holes with no intrastriatal injections. The study will last 5 years, where participants will be blind to treatment allocation for 18 months, followed by an unblinded period of 3.5 years.

The trial has already started recruitment [8], and has a recruitment target of 26 participants, across 4 sites. It will follow a multiple ascending dose design, with a first cohort of 10 participants (stage 2 HD; 6 randomized to low dose and 4 to sham surgery) and a second cohort of 16 participants (stage 1-2 HD; 10 randomized to high dose and 6 to sham surgery).

The primary outcome will be safety, measured at 18 months, and the secondary outcome will be CSF biomarkers, namely levels of the vector DNA and miRNA expression at 60 months. Other outcomes include: biofluid and imaging biomarkers; clinical scales such as the UHDRS motor, cognitive, behaviour and functional subscales, the Huntington's Disease Cognitive Assessment Battery (HD-CAB), 
Table 2

Ongoing pharmacological clinical trials registered at the World Health Organization (WHO) International Clinical Trials Research Platform (ICTRP) for people with Huntington's disease (HD). N/S, not specified; PD, Parkinson's disease; VMAT2, Vesicular Monoamine Transporter 2. Note: IONIS-HTT Rx , ISIS 443139, RG6042 and tominersen refer to the same molecule. New trials since the last Clinical Trials Corner are indicated by *

\begin{tabular}{|c|c|c|c|c|c|c|c|c|c|c|}
\hline Registration ID & Trial name & Intervention & $\begin{array}{l}\text { Mechanism of } \\
\text { Action }\end{array}$ & Population & Comparison & Main outcome & Study design & $\begin{array}{l}\text { Estimated } \\
\text { Enrolment }\end{array}$ & Sponsor & Location \\
\hline NCT04201834* & - & Risperidone & $\begin{array}{l}\text { Dopamine } \\
\text { antagonist }\end{array}$ & $\begin{array}{l}\text { Early and } \\
\text { moderate HD } \\
\text { with chorea }\end{array}$ & None & $\begin{array}{l}\text { Change in } \\
\text { motor scales at } \\
12 \text { weeks }\end{array}$ & $\begin{array}{l}\text { Non- } \\
\text { randomized, } \\
\text { open label } \\
\text { (assessor- } \\
\text { blind), } \\
\text { uncontrolled } \\
\text { trial }\end{array}$ & 12 & $\begin{array}{l}\text { University of } \\
\text { Rochester }\end{array}$ & $\begin{array}{l}\text { USA (single } \\
\text { centre) }\end{array}$ \\
\hline NCT04071639* & - & $\begin{array}{l}\text { Haloperidol, } \\
\text { risperidone, } \\
\text { sertraline and } \\
\text { coenzyme Q10 }\end{array}$ & $\begin{array}{l}\text { Multiple } \\
\text { (dopamine } \\
\text { antagonists, } \\
\text { selective } \\
\text { serotonin } \\
\text { reuptake } \\
\text { inhibitor, } \\
\text { dietary } \\
\text { supplement) }\end{array}$ & $\begin{array}{l}\text { Early and } \\
\text { moderate HD }\end{array}$ & $\begin{array}{l}\text { Coenzyme } \\
\text { Q10 }\end{array}$ & $\begin{array}{l}\text { Efficacy at } 5 \\
\text { years }\end{array}$ & $\begin{array}{l}\text { Randomized, } \\
\text { open label, } \\
\text { controlled, } \\
\text { parallel trial }\end{array}$ & 100 & $\begin{array}{l}\text { Second } \\
\text { Affiliated } \\
\text { Hospital, } \\
\text { School of } \\
\text { Medicine, } \\
\text { Zhejiang } \\
\text { University }\end{array}$ & $\begin{array}{l}\text { China (single } \\
\text { centre) }\end{array}$ \\
\hline NCT04120493* & AMT-130 & $\begin{array}{l}\text { rAAV5- } \\
\text { miHTT }\end{array}$ & $\begin{array}{l}\text { Nonselective } \\
\text { miRNA }\end{array}$ & Early HD & $\begin{array}{l}\text { Sham } \\
\text { intervention }\end{array}$ & $\begin{array}{l}\text { Safety at } 18 \\
\text { months }\end{array}$ & $\begin{array}{l}\text { Randomized, } \\
\text { double-blind, } \\
\text { sham- } \\
\text { controlled, } \\
\text { parallel trial }\end{array}$ & 26 & $\begin{array}{l}\text { UniQure } \\
\text { Biopharma } \\
\text { B.V. }\end{array}$ & $\begin{array}{l}\text { USA } \\
\text { (multi-centre) }\end{array}$ \\
\hline NCT04102579* & KINECT-HD & Valbenazine & $\begin{array}{l}\text { VMAT2 } \\
\text { inhibitor }\end{array}$ & $\begin{array}{l}\text { HD with } \\
\text { chorea }\end{array}$ & Placebo & $\begin{array}{l}\text { Efficacy at } 12 \\
\text { weeks }\end{array}$ & $\begin{array}{l}\text { Randomized, } \\
\text { double-blind, } \\
\text { placebo- } \\
\text { controlled, } \\
\text { parallel trial }\end{array}$ & 120 & $\begin{array}{l}\text { Neurocrine } \\
\text { Biosciences, } \\
\text { Huntington } \\
\text { Study Group }\end{array}$ & $\begin{array}{l}\text { USA } \\
\text { (multi-centre) }\end{array}$ \\
\hline $\begin{array}{l}\text { EUCTR2019- } \\
\text { 002178-30-DK* }\end{array}$ & - & WVE-120102 & $\begin{array}{l}\text { Allele- } \\
\text { selective } \\
\text { antisense } \\
\text { oligonu- } \\
\text { cleotide }\end{array}$ & HD & None & $\begin{array}{l}\text { Safety and } \\
\text { tolerability at } \\
97 \text { weeks }\end{array}$ & $\begin{array}{l}\text { Open-label } \\
\text { extension }\end{array}$ & 70 & $\begin{array}{l}\text { Wave Life } \\
\text { Sciences Ltd. }\end{array}$ & $\begin{array}{l}\text { Australia, } \\
\text { Canada, } \\
\text { Denmark, } \\
\text { France, Poland } \\
\text { and United } \\
\text { Kingdom } \\
\text { (multi-centre) }\end{array}$ \\
\hline
\end{tabular}


Table 2

(Continued)

\begin{tabular}{|c|c|c|c|c|c|c|c|c|c|c|}
\hline Registration ID & Trial name & Intervention & $\begin{array}{l}\text { Mechanism of } \\
\text { Action }\end{array}$ & Population & Comparison & Main outcome & Study design & $\begin{array}{l}\text { Estimated } \\
\text { Enrolment }\end{array}$ & Sponsor & Location \\
\hline NCT04000594* & GEN-PEAK & RG6042 & $\begin{array}{l}\text { Allele- } \\
\text { nonselective } \\
\text { antisense } \\
\text { oligonu- } \\
\text { cleotide }\end{array}$ & HD & None & $\begin{array}{l}\text { Pharmaco- } \\
\text { dynamics and } \\
\text { pharmacoki- } \\
\text { netics at } \\
\text { multiple } \\
\text { timepoints } \\
\text { until } 6 \text { months }\end{array}$ & $\begin{array}{l}\text { Non- } \\
\text { randomized. } \\
\text { open-label, } \\
\text { multiple-dose, } \\
\text { parallel trial }\end{array}$ & 20 & $\begin{array}{l}\text { Hoffmann-La } \\
\text { Roche }\end{array}$ & $\begin{array}{l}\text { The } \\
\text { Netherlands } \\
\text { and UK } \\
\text { (multi-centre) }\end{array}$ \\
\hline NCT03980938* & - & Neflamapimod & $\begin{array}{l}\text { p38 } \alpha \text { MAPK } \\
\text { inhibitor }\end{array}$ & Early HD & Placebo & $\begin{array}{l}\text { Change in } \\
\text { cognitive } \\
\text { scales at } 10 \\
\text { weeks }\end{array}$ & $\begin{array}{l}\text { Randomized, } \\
\text { double-blind, } \\
\text { placebo- } \\
\text { controlled, } \\
\text { cross-over trial }\end{array}$ & 16 & $\begin{array}{l}\text { EIP Pharma } \\
\text { Inc, Voisin } \\
\text { Consulting, } \\
\text { Inc. }\end{array}$ & $\begin{array}{l}\text { UK (single } \\
\text { centre) }\end{array}$ \\
\hline NCT03842969 & GEN-EXTEND & RG6042 & $\begin{array}{l}\text { Allele- } \\
\text { nonselective } \\
\text { antisense } \\
\text { oligonu- } \\
\text { cleotide }\end{array}$ & HD & None & $\begin{array}{l}\text { Safety and } \\
\text { tolerability at } \\
\text { up to } 5 \text { years }\end{array}$ & $\begin{array}{l}\text { Open-label } \\
\text { extension }\end{array}$ & 1050 & $\begin{array}{l}\text { Hoffmann-La } \\
\text { Roche }\end{array}$ & $\begin{array}{l}\text { USA, Canada, } \\
\text { Europe } \\
\text { (multi-centre) }\end{array}$ \\
\hline NCT03761849 & $\begin{array}{l}\text { GENERATION- } \\
\text { HD1 }\end{array}$ & RG6042 & $\begin{array}{l}\text { Allele- } \\
\text { nonselective } \\
\text { antisense } \\
\text { oligonu- } \\
\text { cleotide }\end{array}$ & HD & Placebo & $\begin{array}{l}\text { Clinical } \\
\text { efficacy at } 101 \\
\text { weeks }\end{array}$ & $\begin{array}{l}\text { Randomized, } \\
\text { double-blind, } \\
\text { placebo- } \\
\text { controlled, } \\
\text { parallel trial }\end{array}$ & 909 & $\begin{array}{l}\text { Hoffmann-La } \\
\text { Roche }\end{array}$ & $\begin{array}{l}\text { USA, Canada, } \\
\text { Europe } \\
\text { (multi-centre) }\end{array}$ \\
\hline NCT03515213 & - & Fenofibrate & $\begin{array}{l}\text { PPAR } \alpha \\
\text { agonist }\end{array}$ & HD & Placebo & $\begin{array}{l}\text { Pharmaco- } \\
\text { dynamics at } 6 \\
\text { months }\end{array}$ & $\begin{array}{l}\text { Randomized, } \\
\text { double-blind, } \\
\text { placebo- } \\
\text { controlled, } \\
\text { parallel trial }\end{array}$ & 20 & $\begin{array}{l}\text { University of } \\
\text { California, } \\
\text { Irvine }\end{array}$ & $\begin{array}{l}\text { USA (single } \\
\text { centre) }\end{array}$ \\
\hline NCT03764215 & Tasigna HD & Nilotinib & $\begin{array}{l}\text { Selective } \\
\text { Bcr-Abl } \\
\text { tyrosine kinase } \\
\text { inihbitor }\end{array}$ & HD & None & $\begin{array}{l}\text { Safety, } \\
\text { tolerability } \\
\text { and pharmaco- } \\
\text { dynamics at } 3 \\
\text { months }\end{array}$ & $\begin{array}{l}\text { Open label, } \\
\text { multiple } \\
\text { ascending } \\
\text { dose }\end{array}$ & 20 & $\begin{array}{l}\text { Georgetown } \\
\text { University }\end{array}$ & $\begin{array}{l}\text { USA (single } \\
\text { centre) }\end{array}$ \\
\hline
\end{tabular}




\begin{tabular}{|c|c|c|c|c|c|c|c|c|c|c|}
\hline NCT03225833 & $\begin{array}{l}\text { PRECISION- } \\
\text { HD1 }\end{array}$ & WVE-120101 & $\begin{array}{l}\text { Allele- } \\
\text { selective } \\
\text { antisense } \\
\text { oligonu- } \\
\text { cleotide }\end{array}$ & HD & Placebo & $\begin{array}{l}\text { Safety and } \\
\text { tolerability at } \\
1 \text { and } 120 \text { days }\end{array}$ & $\begin{array}{l}\text { Randomized, } \\
\text { double-blind, } \\
\text { placebo- } \\
\text { controlled, } \\
\text { combined } \\
\text { single } \\
\text { ascending } \\
\text { dose/multiple } \\
\text { ascending } \\
\text { dose trial }\end{array}$ & 48 & $\begin{array}{l}\text { Wave Life } \\
\text { Sciences Ltd. }\end{array}$ & $\begin{array}{l}\text { Australia, } \\
\text { Canada, } \\
\text { Denmark, } \\
\text { France, Poland } \\
\text { and United } \\
\text { Kingdom } \\
\text { (multi-centre) }\end{array}$ \\
\hline NCT03225846 & $\begin{array}{l}\text { PRECISION- } \\
\text { HD2 }\end{array}$ & WVE-120102 & $\begin{array}{l}\text { Allele- } \\
\text { selective } \\
\text { antisense } \\
\text { oligonu- } \\
\text { cleotide }\end{array}$ & HD & Placebo & $\begin{array}{l}\text { Safety and } \\
\text { tolerability at } \\
1 \text { and } 120 \text { days }\end{array}$ & $\begin{array}{l}\text { Randomized, } \\
\text { double-blind, } \\
\text { placebo- } \\
\text { controlled, } \\
\text { combined } \\
\text { single } \\
\text { ascending } \\
\text { dose/multiple } \\
\text { ascending } \\
\text { dose trial }\end{array}$ & 60 & $\begin{array}{l}\text { Wave Life } \\
\text { Sciences Ltd. }\end{array}$ & $\begin{array}{l}\text { Australia, } \\
\text { Canada, } \\
\text { Denmark, } \\
\text { France, Poland } \\
\text { and United } \\
\text { Kingdom } \\
\text { (multi-centre) }\end{array}$ \\
\hline NCT02453061 & TRIHEP 3 & Triheptanoin & $\begin{array}{l}\text { Anaplerotic } \\
\text { therapy }\end{array}$ & HD & Safflower oil & $\begin{array}{l}\text { Pharmaco- } \\
\text { dynamic } \\
\text { efficacy at } 6 \\
\text { months }\end{array}$ & $\begin{array}{l}\text { Randomized, } \\
\text { double-blind, } \\
\text { controlled, } \\
\text { parallel trial }\end{array}$ & 100 & $\begin{array}{l}\text { Institut } \\
\text { National de la } \\
\text { Santé Et de la } \\
\text { Recherche } \\
\text { Médicale, } \\
\text { Ultragenyx } \\
\text { Pharmaceuti- } \\
\text { cal } \\
\text { Inc }\end{array}$ & $\begin{array}{l}\text { France, } \\
\text { Netherlands } \\
\text { (multi-centre) }\end{array}$ \\
\hline NCT02509793 & - & Tetrabenazine & $\begin{array}{l}\text { VMAT2 } \\
\text { inhibitor }\end{array}$ & $\begin{array}{l}\text { HD with } \\
\text { impulsivity }\end{array}$ & None & $\begin{array}{l}\text { Cognitive and } \\
\text { behavioural } \\
\text { effects at } 8 \\
\text { weeks }\end{array}$ & $\begin{array}{l}\text { Single group, } \\
\text { open-label } \\
\text { trial }\end{array}$ & 20 & $\begin{array}{l}\text { University of } \\
\text { Texas Health } \\
\text { Science } \\
\text { Center, and H. } \\
\text { Lundbeck A/S }\end{array}$ & $\begin{array}{l}\text { USA (single } \\
\text { centre) }\end{array}$ \\
\hline
\end{tabular}


Table 2

(Continued)

\begin{tabular}{|c|c|c|c|c|c|c|c|c|c|c|}
\hline Registration ID & Trial name & Intervention & $\begin{array}{l}\text { Mechanism of } \\
\text { Action }\end{array}$ & Population & Comparison & Main outcome & Study design & $\begin{array}{l}\text { Estimated } \\
\text { Enrolment }\end{array}$ & Sponsor & Location \\
\hline NCT02481674 & SIGNAL & VX15/2503 & $\begin{array}{l}\text { Anti- } \\
\text { semaphorin } \\
\text { 4D } \\
\text { monoclonal } \\
\text { antibody }\end{array}$ & $\begin{array}{l}\text { Late } \\
\text { premanifest or } \\
\text { early HD }\end{array}$ & Placebo & $\begin{array}{l}\text { Safety and } \\
\text { tolerability at } \\
15 \text { and } 21 \\
\text { months }\end{array}$ & $\begin{array}{l}\text { Randomized, } \\
\text { double-blind, } \\
\text { placebo- } \\
\text { controlled, } \\
\text { parallel trial }\end{array}$ & 240 & $\begin{array}{l}\text { Vaccinex Inc., } \\
\text { Huntington } \\
\text { Study Group }\end{array}$ & $\begin{array}{l}\text { USA } \\
\text { (multi-centre) }\end{array}$ \\
\hline $\begin{array}{l}\text { EUCTR2013- } \\
\text { 002545-10-SE }\end{array}$ & OSU6162Op & 30Q)-OSU616 & $\begin{array}{l}\text { Monoaminergic } \\
\text { stabilizer }\end{array}$ & $\begin{array}{l}\mathrm{HD}, \mathrm{PD} \text {, brain } \\
\text { trauma, stroke, } \\
\text { myalgic } \\
\text { encephalomyeli- } \\
\text { tis and } \\
\text { narcolepsy }\end{array}$ & None & $\begin{array}{l}\text { Safety at } 3,6 \\
\text { and } 12 \text { months }\end{array}$ & $\begin{array}{l}\text { Single group, } \\
\text { open-label } \\
\text { trial }\end{array}$ & 240 & $\begin{array}{l}\text { A. Carlsson } \\
\text { Research } \mathrm{AB}\end{array}$ & $\begin{array}{l}\text { Sweden } \\
\text { (multi-centre) }\end{array}$ \\
\hline NCT00514774 & UDCA-HD & Ursodiol & Bile acid & HD & Placebo & $\begin{array}{l}\text { Safety, } \\
\text { tolerability } \\
\text { and pharma- } \\
\text { cokinetics at } \\
35 \text { days }\end{array}$ & $\begin{array}{l}\text { Randomized, } \\
\text { double-blind, } \\
\text { placebo- } \\
\text { controlled, } \\
\text { parallel trial }\end{array}$ & 21 & $\begin{array}{l}\text { Oregon Health } \\
\text { and Science } \\
\text { University, } \\
\text { Huntington } \\
\text { Study Group, } \\
\text { Huntington } \\
\text { Society of } \\
\text { Canada }\end{array}$ & $\mathrm{N} / \mathrm{S}$ \\
\hline
\end{tabular}


Table 3

Ongoing invasive non-pharmacological clinical trials registered at the World Health Organization (WHO) International Clinical Trials Research Platform (ICTRP) for people with Huntington's disease (HD). AD, Alzheimer's disease, CBD; Corticobasal Degeneration; DBS, deep brain stimulation; ET, Essential Tremor; GP, Globus pallidus; HT, Holmes Tremor; MNC, mononuclear cells; MS, Multiple Sclerosis; PD, Parkinson's disease; TD, Tardive dyskinesia; WD, Wilson's disease. New trials since the last Clinical Trials Corner are indicated by *

\begin{tabular}{|c|c|c|c|c|c|c|c|c|c|c|}
\hline Registration ID & Trial name & Intervention & $\begin{array}{l}\text { Mechanism } \\
\text { of Action }\end{array}$ & Population & Comparison & Main outcome & Study design & $\begin{array}{l}\text { Esimated } \\
\text { Enrolment }\end{array}$ & Sponsor & Location \\
\hline NCT04244513* & - & GPi DBS & $\begin{array}{l}\text { Deep brain } \\
\text { stimulation }\end{array}$ & $\begin{array}{l}\text { HD with } \\
\text { chorea }\end{array}$ & $\begin{array}{l}\text { Sham } \\
\text { intervention }\end{array}$ & $\begin{array}{l}\text { Efficacy at } 3 \\
\text { and } 6 \text { months }\end{array}$ & $\begin{array}{l}\text { Randomized, } \\
\text { double-blind, } \\
\text { sham- } \\
\text { controlled, } \\
\text { cross-over trial }\end{array}$ & 40 & $\begin{array}{l}\text { Beijing } \\
\text { Municipal } \\
\text { Administration } \\
\text { of Hospitals, } \\
\text { Medtronic }\end{array}$ & $\begin{array}{l}\text { China } \\
\text { (multi- } \\
\text { centre) }\end{array}$ \\
\hline NCT04219241* & ADORE-EXT & Cellavita & $\begin{array}{l}\text { Stem cell } \\
\text { therapy }\end{array}$ & HD & None & $\begin{array}{l}\text { Efficacy and } \\
\text { safety at } 2 \\
\text { years }\end{array}$ & $\begin{array}{l}\text { Open label } \\
\text { extension }\end{array}$ & 35 & $\begin{array}{l}\text { Azidus Brasil, } \\
\text { Cellavita } \\
\text { Pesquisa } \\
\text { Cientifica Ltda }\end{array}$ & $\begin{array}{l}\text { Brazil } \\
\text { (single } \\
\text { centre) }\end{array}$ \\
\hline ISRCTN52651778 & TRIDENT & $\begin{array}{l}\text { Foetal stem } \\
\text { cell transplant }\end{array}$ & $\begin{array}{l}\text { Stem cell } \\
\text { therapy }\end{array}$ & $\begin{array}{l}\text { Early stage } \\
\text { HD }\end{array}$ & Usual care & $\begin{array}{l}\text { Safety at } 4 \\
\text { weeks }\end{array}$ & $\begin{array}{l}\text { Randomized, } \\
\text { open label, } \\
\text { controlled, } \\
\text { parallel trial }\end{array}$ & 30 & $\begin{array}{l}\text { Cardiff } \\
\text { University }\end{array}$ & $\begin{array}{l}\text { UK (single } \\
\text { centre) }\end{array}$ \\
\hline NCT02728115 & SAVE-DH & Cellavita & $\begin{array}{l}\text { Stem cell } \\
\text { therapy }\end{array}$ & HD & None & $\begin{array}{l}\text { Safety at } 5 \\
\text { years }\end{array}$ & $\begin{array}{l}\text { Non- } \\
\text { randomized, } \\
\text { open label, } \\
\text { uncontrolled, } \\
\text { parallel trial }\end{array}$ & 6 & Azidus Brasil & $\begin{array}{l}\text { Brazil } \\
\text { (single } \\
\text { centre) }\end{array}$ \\
\hline NCT03252535 & ADORE-HD & Cellavita & $\begin{array}{l}\text { Stem cell } \\
\text { therapy }\end{array}$ & HD & Placebo & $\begin{array}{l}\text { Efficacy at } 120 \\
\text { days }\end{array}$ & $\begin{array}{l}\text { Randomized, } \\
\text { double-blind, } \\
\text { placebo- } \\
\text { controlled, } \\
\text { parallel trial }\end{array}$ & 35 & Azidus Brasil & $\begin{array}{l}\text { Brazil } \\
\text { (single } \\
\text { centre) }\end{array}$ \\
\hline NCT03297177 & - & $\begin{array}{l}\text { Autologous } \\
\text { stem/stromal } \\
\text { cells }\end{array}$ & $\begin{array}{l}\text { Autologous } \\
\text { stem/stromal } \\
\text { cell injection }\end{array}$ & $\begin{array}{l}\mathrm{HD}, \mathrm{AD}, \mathrm{PD}, \\
\mathrm{CBD}, \mathrm{MS}\end{array}$ & None & $\begin{array}{l}\text { Safety at } 5 \\
\text { years }\end{array}$ & $\begin{array}{l}\text { Single group, } \\
\text { open-label } \\
\text { trial }\end{array}$ & 300 & $\begin{array}{l}\text { Healeon } \\
\text { Medical Inc, } \\
\text { Global Alliance } \\
\text { for Regenerative } \\
\text { Medicine, } \\
\text { Regeneris } \\
\text { Medical }\end{array}$ & $\begin{array}{l}\text { USA and } \\
\text { Honduras } \\
\text { (multi- } \\
\text { centre) }\end{array}$ \\
\hline
\end{tabular}


Table 3

(Continued)

\begin{tabular}{|c|c|c|c|c|c|c|c|c|c|c|}
\hline Registration ID & Trial name & Intervention & $\begin{array}{l}\text { Mechanism } \\
\text { of Action }\end{array}$ & Population & Comparison & Main outcome & Study design & $\begin{array}{l}\text { Esimated } \\
\text { Enrolment }\end{array}$ & Sponsor & Location \\
\hline NCT01834053 & BMACHC & $\begin{array}{l}\text { Bone Marrow } \\
\text { Derived MNC } \\
\text { transplant }\end{array}$ & $\begin{array}{l}\text { Bone marrow } \\
\text { transplant }\end{array}$ & $\begin{array}{l}\text { HD with } \\
\text { chorea }\end{array}$ & None & $\begin{array}{l}\text { Cognitive and } \\
\text { behavioural } \\
\text { effects at } 6 \\
\text { months }\end{array}$ & $\begin{array}{l}\text { Single group, } \\
\text { open-label } \\
\text { trial }\end{array}$ & 50 & $\begin{array}{l}\text { Chaitanya } \\
\text { Hospital, Pune }\end{array}$ & $\begin{array}{l}\text { India (single } \\
\text { centre) }\end{array}$ \\
\hline NCT02252380 & - & $\begin{array}{l}\text { Magnetic } \\
\text { Resonance } \\
\text { Guided } \\
\text { Focused } \\
\text { Ultrasound }\end{array}$ & $\begin{array}{l}\text { Extracranial } \\
\text { stereotactic } \\
\text { radioablation }\end{array}$ & $\begin{array}{l}\text { HD, ET, HT, } \\
\text { PD, WD, } \\
\text { dystonia, TD, } \\
\text { or orofacial } \\
\text { dyskinesias }\end{array}$ & None & $\begin{array}{l}\text { Adverse } \\
\text { events after } \\
\text { the procedure }\end{array}$ & $\begin{array}{l}\text { Single group, } \\
\text { open-label } \\
\text { trial }\end{array}$ & 10 & InSightec & $\begin{array}{l}\text { Canada } \\
\text { (single } \\
\text { centre) }\end{array}$ \\
\hline
\end{tabular}


Table 4

Ongoing non-invasive non-pharmacological clinical trials registered at the World Health Organization (WHO) International Clinical Trials Research Platform (ICTRP) for people with Huntington's disease (HD). AD, Alzheimer's disease; ALS, Amyotrophic Lateral Sclerosis; ET, Essential Tremor; HT, Holmes Tremor; MS, Multiple Sclerosis; PD, Parkinson's disease; TD, Tardive dyskinesia.

\begin{tabular}{|c|c|c|c|c|c|c|c|c|c|c|}
\hline Registration ID & Trial name & Intervention & $\begin{array}{l}\text { Mechanism of } \\
\text { Action }\end{array}$ & Population & Comparison & Main outcome & Study design & $\begin{array}{l}\text { Esimated } \\
\text { Enrolment }\end{array}$ & Sponsor & Location \\
\hline $\begin{array}{l}\text { ACTRN126200 } \\
\text { 00281998* }\end{array}$ & - & Ketogenic diet & - & HD & None & $\begin{array}{l}\text { Change in } \\
\text { cognition and } \\
\text { motor scores at } \\
12 \text { weeks }\end{array}$ & $\begin{array}{l}\text { Non- } \\
\text { randomized, } \\
\text { open label, } \\
\text { single group } \\
\text { trial }\end{array}$ & 10 & $\begin{array}{l}\text { Waikato } \\
\text { Hospital }\end{array}$ & $\begin{array}{l}\text { New } \\
\text { Zealand (-) }\end{array}$ \\
\hline $\begin{array}{l}\text { ACTRN126190 } \\
\text { 00870156* }\end{array}$ & - & $\begin{array}{l}\text { Transcranial } \\
\text { alternating } \\
\text { current } \\
\text { stimulation }\end{array}$ & $\begin{array}{l}\text { Transcranial } \\
\text { magnetic } \\
\text { stimulation }\end{array}$ & $\begin{array}{l}\text { Premanifest } \\
\text { and early HD }\end{array}$ & $\begin{array}{l}\text { Sham } \\
\text { intervention }\end{array}$ & Biomarkers & $\begin{array}{l}\text { Randomized, } \\
\text { open-label, } \\
\text { cross-over } \\
\text { trials }\end{array}$ & 60 & $\begin{array}{l}\text { Monash } \\
\text { University, } \\
\text { Epworth } \\
\text { Centre for } \\
\text { Innovation in } \\
\text { Mental Health }\end{array}$ & $\begin{array}{l}\text { Australia } \\
\text { (single } \\
\text { centre) }\end{array}$ \\
\hline $\begin{array}{l}\text { ACTRN126180 } \\
01717246\end{array}$ & - & $\begin{array}{l}\text { Multidisci- } \\
\text { plinary therapy } \\
\text { program }\end{array}$ & $\begin{array}{l}\text { Exercise, } \\
\text { cognitive } \\
\text { training, } \\
\text { lifestyle } \\
\text { guidance and } \\
\text { social } \\
\text { activities }\end{array}$ & $\begin{array}{l}\text { Premanifest } \\
\text { HD }\end{array}$ & $\begin{array}{l}\text { Standard of } \\
\text { care }\end{array}$ & $\begin{array}{l}\text { Feasibility and } \\
\text { safety }\end{array}$ & $\begin{array}{l}\text { Clustered, } \\
\text { non- } \\
\text { randomized, } \\
\text { open label, } \\
\text { parallel trial }\end{array}$ & 40 & $\begin{array}{l}\text { Edith Cowan } \\
\text { University, } \\
\text { Deakin } \\
\text { University and } \\
\text { Lotterywest }\end{array}$ & $\begin{array}{l}\text { Australia } \\
\text { (two centres) }\end{array}$ \\
\hline NCT03417583 & - & $\begin{array}{l}\text { Neuropsy- } \\
\text { chiatric } \\
\text { treatment } \\
\text { protocol }\end{array}$ & $\begin{array}{l}\text { Multidisci- } \\
\text { plinary } \\
\text { intervention }\end{array}$ & $\begin{array}{l}\text { HD with neu- } \\
\text { ropsychiatric } \\
\text { symptoms }\end{array}$ & $\begin{array}{l}\text { Standard of } \\
\text { care }\end{array}$ & $\begin{array}{l}\text { Change in } \\
\text { quality of life at } \\
18 \text { months }\end{array}$ & $\begin{array}{l}\text { Non- } \\
\text { randomized, } \\
\text { assessor- } \\
\text { blinded, } \\
\text { parallel trial }\end{array}$ & 100 & $\begin{array}{l}\text { Vanderbilt } \\
\text { University } \\
\text { Medical } \\
\text { Center and } \\
\text { Teva Pharma- } \\
\text { ceuticals } \\
\text { USA }\end{array}$ & $\begin{array}{l}\text { USA (single } \\
\text { centre) }\end{array}$ \\
\hline $\begin{array}{l}\text { CTRI/2018/01/ } \\
011359\end{array}$ & - & $\begin{array}{l}\text { Repetitive } \\
\text { transcranial } \\
\text { magnetic } \\
\text { stimulation }\end{array}$ & $\begin{array}{l}\text { Transcranial } \\
\text { magnetic } \\
\text { stimulation }\end{array}$ & $\begin{array}{l}\text { Early to } \\
\text { moderate HD } \\
\text { and PD }\end{array}$ & $\begin{array}{l}\text { Sham } \\
\text { stimulation }\end{array}$ & $\begin{array}{l}\text { Efficacy at } 5 \\
\text { days }\end{array}$ & $\begin{array}{l}\text { Randomized, } \\
\text { single-blind, } \\
\text { placebo- } \\
\text { controlled, } \\
\text { parallel trial }\end{array}$ & 40 & Vinay Goyal & $\begin{array}{l}\text { India (single } \\
\text { centre) }\end{array}$ \\
\hline
\end{tabular}


Table 4

(Continued)

\begin{tabular}{|c|c|c|c|c|c|c|c|c|c|c|}
\hline Registration ID & Trial name & Intervention & $\begin{array}{l}\text { Mechanism of } \\
\text { Action }\end{array}$ & Population & Comparison & Main outcome & Study design & $\begin{array}{l}\text { Esimated } \\
\text { Enrolment }\end{array}$ & Sponsor & Location \\
\hline NCT03344601 & PACE-HD & $\begin{array}{l}\text { Supported } \\
\text { structured } \\
\text { aerobic exercise } \\
\text { training program }\end{array}$ & Physiotherapy & HD & $\begin{array}{l}\text { Activity as } \\
\text { usual }\end{array}$ & $\begin{array}{l}\text { Data } \\
\text { completeness, } \\
\text { recruitment, } \\
\text { retention, safety, } \\
\text { adherence, } \\
\text { fidelity and } \\
\text { acceptability at } \\
12 \text { months }\end{array}$ & $\begin{array}{l}\text { Nested } \\
\text { open-label, } \\
\text { randomized } \\
\text { controlled } \\
\text { parallel trial }\end{array}$ & 120 & $\begin{array}{l}\text { Cardiff } \\
\text { University and } \\
\text { CHDI } \\
\text { Foundation, } \\
\text { Inc }\end{array}$ & $\begin{array}{l}\text { Germany, } \\
\text { Spain and } \\
\text { USA (multi- } \\
\text { centre) }\end{array}$ \\
\hline $\begin{array}{l}\text { ACTRN126170 } \\
01269325\end{array}$ & - & $\begin{array}{l}\text { Swallowing skill } \\
\text { training }\end{array}$ & $\begin{array}{l}\text { Speech and } \\
\text { language } \\
\text { therapy }\end{array}$ & HD and ALS & None & $\begin{array}{l}\text { Swallowing } \\
\text { function and } \\
\text { quality of life at } \\
2 \text { weeks }\end{array}$ & $\begin{array}{l}\text { Single group, } \\
\text { open-label } \\
\text { trial }\end{array}$ & 54 & $\begin{array}{l}\text { University of } \\
\text { Canterbury }\end{array}$ & $\begin{array}{l}\text { New } \\
\text { Zealand } \\
\text { (single } \\
\text { centre) }\end{array}$ \\
\hline
\end{tabular}


the Neuro-QoL, HDQLIFE and Hospital Anxiety and Depression Scale (HADS); and quantitate motor assessments (i.e. Q-Motor).

Sponsors/funders: UniQure Biopharma B.V..

Comments: The AAV5-miHTT is an engineered microRNA (miRNA) targeting both human wild-type and mutant huntingtin for degradation. It is delivered via an adeno-associated viral vector serotype 5 (AAV5). This is the first human trial of an AAVmediated gene therapy in Huntington's disease.

If it functions as intended, upon injection into the brain parenchyma using MRI-guided convectionenhanced delivery, the AAV5-miHTT will bind to cell receptors and will be internalised by neurons and transported to the nucleus. There, the miRNA will be uncoated from the viral vector and remains episomal. After expression and processing of the miHTT transgene by the endogenous RNA interference machinery into a hairpin structure, the miRNA is transported into the cytoplasm. There the mature miRNA will load in the RNA-induced silencing complex and bind huntingtin mRNA, targeting it for cleavage and degradation. In theory, this mechanism of action makes this method irreversible, and animal models have demonstrated long-lasting miRNA expression over time after a single injection.

The efficacy and safety of this miRNA and vector has been assessed in cultured human neurons, and in vivo in multiple animal models such as mice, nonhuman primates and transgenic minipigs. Transgene expression accompanied by huntingtin lowering has been seen in the injected and distant structures such as the cortex.

The selected vector - AAV5 - has been tested in 4 clinical studies across haematological and metabolic disorders. When given intravenously it appears safe and tolerable, showing low activity to pre-existing neutralizing antibodies. However this is the first time it has been used for intraparenchymal delivery into the brain.

The AAV5-miHTT will be injected to the caudate and putamen bilaterally via MRI-guided convectionenhanced delivery. This approach involves surgical exposure of the brain tissue, and insertion of small diameter catheters into the injected structures. Injection usually takes long time periods (several hours) and a pressure gradient in order to saturate the targeted tissues. Even with these techniques, there is limited tissue distribution after injection. In non-human models both the vector and huntingtin lowering have been demonstrated to be present in distant structures, such as the cortex. It is unclear whether this occurs via axonal transport or by some other mechanism such as secretion and absorption of miRNA-containing exosomes.

This is a challenging trial using a novel therapeutic approach. The community will be looking forward to learning more about the feasibility of the approach, its safety, and efficacy.

\section{KINECT-HD (NCT04102579)}

Study title: A Phase 3, Randomized, Double-Blind, Placebo-Controlled Study to Assess the Efficacy, Safety, and Tolerability of Valbenazine for the Treatment of Chorea Associated With Huntington Disease [2].

Intervention: Once daily valbenazine, [9] a VMAT2 inhibitor.

Description: The KINECT-HD trial, sponsored by Neurocrine Biosciences and the Huntington Study Group, aims to evaluate the efficacy, safety and tolerability of valbenazine in adults (18 to 75 years of age) with a clinical diagnosis of HD with chorea, compared with placebo. The purpose is to assess whether valbenazine is more effective than placebo in reducing chorea associated with HD.

Individuals with the following are not eligible: a history of prior VMAT2 inhibitor therapy; swallowing difficulties; who are pregnant or breastfeeding; or with a history of long QT syndrome, cardiac tachyarrhythmia, left bundle-branch block, atrioventricular block, bradycardia or hear failure; unstable or serious medical or psychiatric illness; significant suicidal risk; substance dependence or abuse; unstable antidepressant regimen; previous history of gene therapy; receiving an investigational drug within 30 days of baseline visit; and blood donation or significant blood loss $(\geq 550 \mathrm{~mL})$ within 30 days of baseline visit.

KINECT-HD is an international, multi-centre, randomized, double-blind, controlled, parallel phase 3 trial. It has 2 study arms: the active group, where participants will receive valbenazine once daily up to $80 \mathrm{mg}$ based on tolerability for 12 weeks; and the comparator group, where participants will receive a placebo capsule once daily for 12 weeks.

The study will last around 15 weeks, with an 8week dose adjustment (i.e. $40 \mathrm{mg}>60 \mathrm{mg}>80 \mathrm{mg}$ ) based on tolerability followed by 4 weeks of dose 
maintenance, and will enrol 120 participants equally distributed across groups. Recruitment is currently ongoing, and approximately 55 centres across the US and Canada will be involved.

The primary outcome measure is change in chorea at 12 weeks measured as a sum of the chorea items of the UHDRS Total Motor Score. Secondary outcomes include subjective impression of change; quality of life and digital biomarkers.

Sponsors/funders: Neurocrine Biosciences and the Huntington Study Group.

Comments: Valbenazine is a vesicular monoamine transporter 2(VMAT2) inhibitor previously approved by FDA for tardive dyskinesia. It is a prodrug of dihydrotetrabenazine that reduces dopamine release into the synaptic cleft by selectively inhibiting presynaptic VMAT2.

There are two other VMAT2 inhibitors on the market: tetrabenazine (3-times daily) and deutatrabenazine (twice-daily), both of them approved by FDA for chorea associated with HD. Apart from dosage regimen, it is unclear if there are differences between these two modestly effective drugs, which have comparable safety profiles with risks of suicidality, parkinsonism and QT prolongation [10-12].

Currently, valbenazine is FDA-approved for tardive dyskinesia ( $40 \mathrm{mg}$ daily for one week followed by $80 \mathrm{mg}$ daily thereafter) and has had an unsuccessful trial in paediatric Tourette's syndrome.

\section{ACKNOWLEDGMENTS}

The authors are supported by CHDI Foundation, Inc. (salary support to FBR and EJW for conduct of the HDClarity study).

\section{CONFLICTS OF INTEREST}

FBR and EJW were sub-investigators on LEGATO-HD (NCT02215616), IONIS HTT $_{\mathrm{Rx}}$ (NCT02519036) and IONIS HTT Rx $_{\text {OLE (NCT03 }}$ 342053), are sub-investigators on the Roche GENERATION-HD (NCT03761849), Roche Natural History Study (NCT03664804) and Roche GEN-EXTEND (NCT03842969) trials, and EJW was a sub-investigator on the Amaryllis (NCT02197130). EJW is the chief investigator of the Roche GEN-PEAK trial (NCT04000594) and FBR is a sub-investigator. The authors did not make use of confidential or privileged information: all materials included in this manuscript were collected from publicly available sources. FBR has provided consultancy services to GLG. EJW has participated in scientific advisory boards with Hoffmann-La Roche Ltd, Ionis, Shire, GSK, Wave Life Sciences, PTC Therapeutics, Takeda and Mitoconix. All honoraria were paid through UCL Consultants Ltd, a wholly owned subsidiary of UCL. Their Host Institution, University College London Hospitals NHS Foundation Trust, has received funds as compensation for conducting clinical trials for Ionis Pharmaceuticals, Pfizer and Teva Pharmaceuticals. Hoffman La Roche Ltd has supported UCL with research funding for EJW.

\section{REFERENCES}

[1] UniQure Biopharma B.V. Safety and Proof-of-Concept (POC) Study With AMT-130 in Adults With Early Manifest Huntington Disease. https://ClinicalTrials.gov/ show/NCT04120493; 2019.

[2] Neurocrine Biosciences, Huntington Study Group. Efficacy, Safety, and Tolerability of Valbenazine for the Treatment of Chorea Associated With Huntington Disease. https://ClinicalTrials.gov/show/NCT04102579; 2019.

[3] Rodrigues FB, Wild EJ. Clinical Trials Corner: September 2017. J Huntingtons Dis. 2017;6(3):255-63.

[4] Wave Life Sciences Ltd. Safety and Tolerability of WVE-120102 in Patients With Huntington's Disease. https://ClinicalTrials.gov/show/NCT03225846; 2017.

[5] Wave Life Sciences Ltd. Safety and Tolerability of WVE-120101 in Patients With Huntington's Disease. https://ClinicalTrials.gov/show/NCT03225833; 2017.

[6] Wave Life Sciences Announces Topline Data and Addition of Higher Dose Cohort in Ongoing Phase 1b/2a PRECISION-HD2 Trial in Huntington's Disease [press release]. 2019.

[7] Miniarikova J, Evers MM, Konstantinova P. Translation of MicroRNA-Based Huntingtin-Lowering Therapies from Preclinical Studies to the Clinic. Mol Ther. 2018;26(4):94762.

[8] uniQure Announces Third Quarter 2019 Results and Highlights Recent Company Progress [press release]. https:// tools.eurolandir.com/tools/Pressreleases/GetPressRelease/ $? \mathrm{ID}=3658637 \&$ lang $=$ en-GB $\&$ companycode $=$ nl-qure $\& \mathrm{v}=$ 2019.

[9] Mochel F. Triheptanoin for the treatment of brain energy deficit: A 14-year experience. Journal of Neuroscience Research. 2017;95(11):2236-43.

[10] Rodrigues FB, Duarte GS, Costa J, Ferreira JJ, Wild E. Meta-research metrics matter: Letter regarding article "Indirect tolerability comparison of Deutetrabenazine and Tetrabenazine for Huntington disease". J Clin Mov Disord. 2017;4(9):1-3.

[11] Rodrigues FB, Duarte GS, Costa J, Ferreira JJ, Wild EJ. Tetrabenazine Versus Deutetrabenazine for Huntington's Disease: Twins or Distant Cousins? Movement Disorders Clinical Practice. 2017;4(4):582-5. 
[12] Claassen DO, Carroll B, De Boer LM, Wu E, Ayyagari R, Gandhi S, et al. Indirect tolerability comparison of Deutetrabenazine and Tetrabenazine for Huntington disease. J Clin Mov Disord. 2017;4:3.

[13] Rodrigues FB, Wild EJ. Huntingtons Disease Clinical Trials Corner: February 2018. Journal of Huntington's Disease. 2018;7(1):89-98.

[14] Rodrigues FB, Wild EJ. Huntington's Disease Clinical Trials Corner: August 2018. J Huntingtons Dis. 2018;7(3):279-86.
[15] Rodrigues FB, Quinn L, Wild EJ. Huntington's Disease Clinical Trials Corner: January 2019. Journal of Huntington's Disease. 2019;8(1):115-25.

[16] Rodrigues FB, Ferreira JJ, Wild EJ. Huntington's Disease Clinical Trials Corner: June 2019. J Huntingtons Dis. 2019;8(3):363-71. 